University of Nebraska - Lincoln

DigitalCommons@University of Nebraska - Lincoln

June 2006

\title{
Laser acceleration of electrons to giga-electron-volt energies using highly charged ions
}

S.X. Hu

University of Nebraska - Lincoln

Anthony F. Starace

University of Nebraska-Lincoln, astarace1@unl.edu

Follow this and additional works at: https://digitalcommons.unl.edu/physicsstarace

Part of the Physics Commons

Hu, S.X. and Starace, Anthony F., "Laser acceleration of electrons to giga-electron-volt energies using highly charged ions" (2006). Anthony F. Starace Publications. 86.

https://digitalcommons.unl.edu/physicsstarace/86

This Article is brought to you for free and open access by the Research Papers in Physics and Astronomy at DigitalCommons@University of Nebraska - Lincoln. It has been accepted for inclusion in Anthony F. Starace Publications by an authorized administrator of DigitalCommons@University of Nebraska - Lincoln. 


\title{
Laser acceleration of electrons to giga-electron-volt energies using highly charged ions
}

\author{
S. X. Hu* and Anthony F. Starace ${ }^{\dagger}$ \\ Department of Physics and Astronomy, The University of Nebraska, Lincoln, Nebraska 68588-0111, USA
}

(Received 13 June 2005; revised manuscript received 31 March 2006; published 7 June 2006)

\begin{abstract}
The recent proposal to use highly charged ions as sources of electrons for laser acceleration [S. X. Hu and A. F. Starace, Phys. Rev. Lett. 88, 245003 (2002)] is investigated here in detail by means of three-dimensional, relativistic Monte Carlo simulations for a variety of system parameters, such as laser pulse duration, ionic charge state, and laser focusing spot size. Realistic laser focusing effects—e.g., the existence of longitudinal laser field components-are taken into account. Results of spatial averaging over the laser focus are also presented. These numerical simulations show that the proposed scheme for laser acceleration of electrons from highly charged ions is feasible with current or near-future experimental conditions and that electrons with $\mathrm{GeV}$ energies can be obtained in such experiments.
\end{abstract}

DOI: 10.1103/PhysRevE.73.066502

PACS number(s): 41.75.Jv, 52.38.Kd, 32.80.Fb, 52.65.Pp

\section{INTRODUCTION}

Technological advances over the past two decades (e.g., the invention of chirped pulse amplification [1]) have led to great increases in laser intensities. Recently, petawatt $\left(10^{15} \mathrm{~W}\right)$ lasers have become available [2], making possible laser intensities of $\sim 10^{22} \mathrm{~W} / \mathrm{cm}^{2}$ and higher [2-7]. The laser field strengths corresponding to these intensities may reach $\sim 10^{12} \mathrm{~V} / \mathrm{cm}$, which is nearly three orders of magnitude greater than the Coulomb field that binds the ground-state electron in atomic hydrogen. Many novel phenomena resulting from the interaction of such superstrong lasers with matter are currently being investigated [8-11]. Among the key features of this interaction are the production of highly charged ions as well as of fast charged particles, including electrons. For example, highly charged ions have been observed from clusters irradiated by intense laser pulses $[12,13]$. Furthermore, these ions have been found to be quite energetic $[14,15]$. Also, intense laser interactions with solid targets have resulted in the production of fast protons with energies in the range of $1-18 \mathrm{MeV}[16,17]$. Investigations of electron acceleration by such huge laser fields was explored intensively both theoretically and experimentally beginning in the 1960s and 1970s (see, e.g., [18]), simultaneously with the technological strides that were leading to great increases in laser intensities [4].

Plasma-based electron acceleration schemes were among the first to be studied. In 1979 Tajima and Dawson [19] proposed using laser beams to excite plasma wakefields for electron acceleration. They indicated that the wakefields would be particularly large if the laser pulse duration is of the order of the plasma period (the so-called "laser wakefield accelerator"); they also proposed a scheme involving two laser beams having a frequency difference equal to the plasma frequency (the so-called "plasma beat wave accelerator"). Recently these and other proposals for plasma-based particle accelerators have been reviewed [20]. Numerous experi-

\footnotetext{
*Present address: Laboratory for Laser Energetics, University of Rochester, 250 East River Road, Rochester, NY 14623.

†Electronic address: astarace1@unl.edu
}

ments have confirmed such plasma-based accelerator concepts [21-28]. Very recently, great advances have been reported in obtaining nearly monochromatic beams of electrons with energies in the range from $50 \mathrm{MeV}$ to $170 \mathrm{MeV}$ with excellent collimation [29-31]. Whether or not electrons can be accelerated to $\mathrm{GeV}$ energies by one of the plasma-based schemes, however, remains an open question. Theoretical analyses by Sprangle et al. [32] of the laser wakefield acceleration of electrons in plasma channels have predicted electron energies of the order of $\mathrm{GeV}$; however, this can only be achieved provided the intense laser pulse can propagate a long enough distance in the plasma without disruption. Note that for laser acceleration schemes based on plasma waves there are very exacting conditions for injecting electrons into extremely short and narrow acceleration buckets [33-35]. Also, the field strength within a laser-induced wakefield is generally smaller than the laser field strength itself.

Alternatively, electrons in vacuum may be accelerated by the laser field directly. This acceleration mechanism has also been investigated both theoretically [36-39] and experimentally $[40,41]$. The major difficulty in accelerating free electrons is that they are expelled from the laser focal region before seeing the maximum amplitude of a laser pulse (see, e.g., [42]). This difficulty may be addressed by accelerating electrons sequentially and cumulatively by more than one laser accelerator, as demonstrated successfully recently by Kimura et al. [43] for the case of two serial laser accelerators acting on an electron beam in vacuum. Alternatively, an already fast electron beam can be injected into the highestintensity region of the laser focus in order to accelerate the electrons to even higher energies, as proposed recently by Salamin and Keitel [44,45]. As their calculations for the case of tightly focused petawatt lasers show, the electrons need an initial kinetic energy of the order of a few $\mathrm{MeV}$ in order to overcome the ponderomotive potential and penetrate inside the laser focal region, whereupon they may be accelerated by the laser to $\mathrm{GeV}$ energies. However, since high laser intensities in the focal region require not only a tight focus but also a short pulse duration, timing the coincidence of the electron beam with the laser pulse is important. Also, the final electron energies are very sensitive both to the angle of injection of the electrons with respect to the laser propagation axis and 
also to the point of injection of the electrons with respect to the laser focus $[44,45]$.

An alternative to accelerating free electrons is to accelerate initially bound electrons, as these will experience a higher laser intensity once they are free (i.e., ionized) than would initially free electrons. Theoretically, hot electrons with $\mathrm{MeV}$ energies have been predicted for neutral atom targets [46]. Experimentally, electrons with energies of a few $\mathrm{keV}$ have been observed for cluster targets [47]. Both of these results for the final electron energies are well below the expected $\mathrm{GeV}$ energies that are in principle possible with currently available peak laser intensities, thereby indicating that electrons ionized from neutral targets are "born" in the laser field prematurely, before the laser pulse has reached its maximum intensity.

To overcome the difficulties inherent to laser acceleration of either free electrons or electrons bound in neutral targets, we recently proposed using highly charged ions as targets for laser acceleration of electrons to $\mathrm{GeV}$ energies [42]. Owing to the practical impossibility of solving the threedimensional Dirac equation for the electron motion (because of the extremely large-scale, laser-driven electron trajectories), we employed instead a three-dimensional classical, relativistic Monte Carlo approach. (For a brief review of Monte Carlo methods in intense laser physics, see Sec. 5.4 of Ref. [9].) Our simulations showed that since an electron in a highly charged ion is very tightly bound, it remains bound (with high probability) during the rise time of an intense laser pulse. For an appropriately chosen highly charged ion, the bound electron only becomes ionized when the laser pulse intensity reaches its maximum, whereupon it is accelerated to $\mathrm{GeV}$ energies by the peak laser electric field amplitude. These predictions were confirmed in a similar theoretical study by Maltsev and Ditmire [48] using a different, semiclassical Monte Carlo method. Their simulations [48] employed a much more tightly focused laser field than in the simulations of Ref. [42] in order to demonstrate the effects on the ionized electron spectrum of a breakdown of the paraxial approximation-i.e., the effects of longitudinal electric fields in the focal region [37,49-52]. Their results showed that the longitudinal electric fields reduce the maximum kinetic energies of the accelerated electrons and lead to a much wider spread of emission angles at any given kinetic energy. One may thus conclude that the laser beam waist in the focal region is another parameter upon which the energy and angular distributions of accelerated electrons must be optimized; the tighter the focus, the greater the transverse laser fields that can ionize electrons, but also the greater the longitudinal fields that may work against such acceleration.

In this paper, we analyze in detail the scheme for accelerating electrons to $\mathrm{GeV}$ energies that we proposed in Ref. [42], which involves highly charged ions as targets. We also survey the dependence of this laser acceleration scheme on both laser and target ion parameters. In particular, we take into account higher-order corrections to the paraxial approximation in the laser focal region [37,48-52]. Our threedimensional, classical relativistic Monte Carlo simulations show that electrons bound in highly charged ions can be accelerated to $\mathrm{GeV}$ energies for a wide range of laser, target ion, and focal parameters.
This paper is organized as follows: In Sec. II A, we briefly review the three-dimensional, classical relativistic Monte Carlo simulation employed in our calculations. In Sec. II B we describe the relativistic microcanonical ensemble employed to represent the initial state of our highly charged ion target system. In Sec. II C, we review the corrections to the paraxial approximation required to describe the laser fields in the case of tight focusing-i.e., when the beam waist is comparable to the carrier wavelength. In Sec. III, we present results of our three-dimensional, classical relativistic Monte Carlo simulations for different sets of laser, target, and focal parameters. We begin this section with the illustrative case of free electrons as targets, which indicates the necessity of using highly charged ion targets. We then analyze the dependence of the ionized electron energy and angular distributions on the laser beam waist, laser pulse duration, laser intensity, and ion charge state. Finally, we examine the spatial distribution of the ionized electrons relative to the laser propagation axis. In Sec. IV we summarize our results and present our conclusions.

\section{SIMULATION METHOD AND TREATMENT OF LASER FOCUSING EFFECTS}

Relativistic interactions of superstrong laser fields with ions have recently been the subject of numerous theoretical studies [53-59]. For a tightly bound system, it is possible to solve quantum mechanically either the weakly relativistic Schrödinger equation $[54,55,59]$ or the Dirac $[57,58]$ equation for the inner atomic dynamics because the electron wave packet is well confined by the strong ionic core. However, it is extremely difficult and time consuming to investigate quantum mechanically the relativistic motion of an ionized electron wave packet because the excursions of a free electron in a superstrong laser field are enormous (on an atomic scale), attaining even macroscopic magnitudes. Thus, classical Monte Carlo [42] or semiclassical methods [48,60] are usually employed for such studies. These classical methods involve a microcanonical ensemble that mimics the quantum-mechanical ground state of interest. This so-called "phase-space-averaging" method [61] was originally developed to study microwave ionization of Rydberg atoms. It was first applied to laser-atom multiphoton ionization processes in 1987 [62,63]. Since then, it has been employed in studies of a variety of intense laser-atom processes (see, e.g., the review by Protopapas et al. [9]). The justification of the method for intense laser processes has been discussed in detail by Gajda et al. [64]. Also, for the highest laser intensities, relativistic versions of the method have been developed $[46,65]$. Our simulations presented here are carried out using the three-dimensional, relativistic, classical Monte Carlo method. In this section, we describe first the interaction scheme and review the relativistic Monte Carlo method. We then outline the procedure for preparing a relativistic microcanonical ensemble of initial states. Finally, in the last subsection we describe our treatment of tight focusing effects on the laser fields.

\section{A. Interaction scheme and the relativistic Monte Carlo method}

We consider the interaction of an ultraintense laser pulse with a hydrogenlike, highly charged ion. Figure 1 shows the 


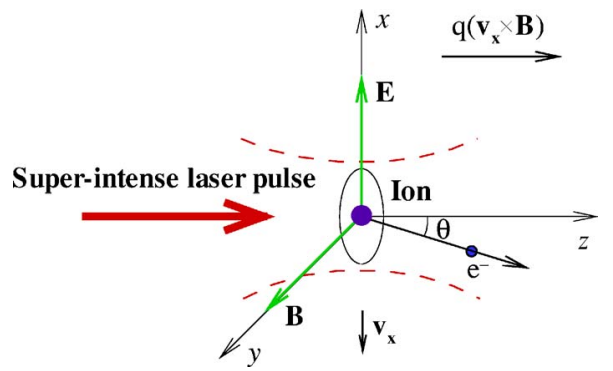

FIG. 1. (Color online) Schematic diagram of the interaction of an intense laser pulse with a hydrogenlike, highly charged ion that results in ionization of its electron. The electric field $E$ and the magnetic field $B$ of the intense laser pulse are linearly polarized along the $x$ and $y$ axes respectively; the laser pulse propagates along the $z$ axis. The angle $\theta$ defines the electron ejection angle with respect to the laser propagation axis ( $z$ direction). The vector $\mathbf{v}_{\mathbf{x}}$ denotes the electron velocity component along the $x$ axis, and $q$ $=-e$ denotes the electron charge. The component of the Lorentz force along the $z$ axis is indicated explicitly in the upper right of the figure.

interaction scheme. The laser pulse is assumed to be linearly polarized along the $x$ axis and to propagate along the $z$ axis; it is focused to a small spot having a radius (or beam waist $w_{0}$ ) on the order of $10 \mu \mathrm{m}$. While the laser field components in the focal region are analyzed in the last subsection (see below), we note here that for the case of tight focusing the laser field has components along all three axes in the focal region, even though it is mainly polarized along the $x$ axis (as indicated in Fig. 1). The relativistic classical dynamics of an electron in both an electromagnetic field and a Coulomb field is described by

$$
\begin{gathered}
d \mathbf{r} / d t=\mathbf{p} / \gamma, \\
d \mathbf{p} / d t=-\left(\mathbf{E}_{L}+\mathbf{E}_{C}+\mathbf{p} \times \mathbf{B}_{L} / \gamma c\right),
\end{gathered}
$$

where $\gamma \equiv \sqrt{1+\mathbf{p}^{2} / c^{2}}$ is the usual relativistic factor; $c$ $\simeq 137.036$ a.u. is the speed of light in vacuum; $\mathbf{r}$ and $\mathbf{p}$ are the coordinate and mechanical momentum vectors of the electron, respectively; $\mathbf{E}_{L}$ is the laser electric field; and the Coulomb electric field of the nucleus is denoted by $\mathbf{E}_{C}$ $=-\nabla V(r)$, where $V(r)$ is the three-dimensional nuclear Coulomb potential. [Note that atomic units (a.u.)-i.e., $e=m_{e}$ $=\hbar=1$-are used throughout this paper, although we give most laser and ion parameters also in SI units; in particular, 1 a.u. of electric field amplitude equals 5.142208 $\times 10^{9} \mathrm{~V} / \mathrm{cm}$.] The physics embodied within the above vector equations may be more easily discerned by writing them in terms of their three components,

$$
\begin{gathered}
d x / d t=p_{x} / \gamma, \\
d p_{x} / d t=-\left[E_{L x}+E_{C x}+\left(p_{y} B_{L z}-p_{z} B_{L y}\right) / \gamma c\right], \\
d y / d t=p_{y} / \gamma, \\
d p_{y} / d t=-\left[E_{L y}+E_{C y}+\left(p_{z} B_{L x}-p_{x} B_{L z}\right) / \gamma c\right],
\end{gathered}
$$

$$
\begin{gathered}
d z / d t=p_{z} / \gamma, \\
d p_{z} / d t=-\left[E_{L z}+E_{C z}+\left(p_{x} B_{L y}-p_{y} B_{L x}\right) / \gamma c\right],
\end{gathered}
$$

where, e.g., $(x, y, z)$ are the three components of the coordinate vector $\mathbf{r}$ and similarly for the other vectors. The main electromagnetic field components of the linearly polarized laser are $E_{L x}$ and $B_{L y}$. From these equations, we see that along the $x$ direction the major component of the Lorentz force, $p_{z} B_{L y} / \gamma c$, is directed opposite to the electric force $-E_{L x}$, while along the $z$ direction the major component of the Lorentz force, $-p_{x} B_{L y} / \gamma c$, accelerates the electron along the laser propagation direction.

Using the Runge-Kutta method with variable step size, we integrate Eqs. (2) numerically for each classical electron trajectory. The electron trajectories are chosen randomly from our preprepared relativistic microcanonical ensemble, which mimics the electronic ground state of the hydrogenlike, highly charged ion target. We trace a sufficient number of trajectories until statistically unchanged results are obtained. We define $\theta=\cos ^{-1}\left(p_{z} /|p|\right)$ as the electron emission angle between the electron ejection direction and the laser propagation direction (i.e., the $z$ axis), as shown in Fig. 1. Our results are presented in the next section as plots of the accelerated electron's final-state energy as a function of this emission angle $\theta$.

\section{B. Preparation of a relativistic microcanonical ensemble}

In order to integrate the above equations (2), we require for each trajectory an initial set of parameters. In fact, we must prepare a microcanonical ensemble of such parameters that collectively mimic the ground state of the quantum system under consideration, which in this paper is a hydrogenlike, highly charged ion. The standard procedure for preparing such a relativistic microcanonical ensemble involves two main steps $[61,65,66]$.

\section{First step: Planar ensemble}

Since $\mathbf{r} \times \mathbf{p}$ is a constant vector, the electron motion for any particular electron trajectory in the Coulomb potential of the highly charged ion must be planar (in the absence of the laser field). It is most convenient to use polar coordinates $(r, \phi)$ in that plane, chosen here to be the $x-y$ plane, with the origin at the position of the nucleus. We solve the following relativistic Kepler problem [67] for an angular momentum $L=r \times p$ and a total energy $E_{0}=\varepsilon+c^{2}$ (where $\varepsilon$ represents the quantum ground-state energy of the system, whose value is negative, and where $c^{2}$ is the rest energy of the electron):

$$
1+\frac{L^{2}}{c^{2}}\left[\left(\frac{1}{r^{2}} \frac{d r}{d \phi}\right)^{2}+\frac{1}{r^{2}}\right]=\left(\frac{E_{0}+Z / r}{c^{2}}\right)^{2}
$$

With the substitution $s=\frac{1}{r}$, the relativistic Kepler equation becomes 


$$
\frac{d^{2} s}{d \phi^{2}}+\lambda^{2} s=D
$$

with $\lambda^{2}=1-Z^{2} / c^{2} L^{2}$ and $D=Z E_{0} / c^{2} L^{2}$. (Note that $\lambda$ approaches unity in the nonrelativistic limit-i.e., $c \rightarrow \infty$.) The solution of Eq. (4) can be expressed as

$$
s=\frac{1}{r}=A \cos (\lambda \phi)+B \sin (\lambda \phi)+D / \lambda^{2},
$$

where $A$ and $B$ are constants of integration, which are to be determined. We choose the line from which $\phi$ is measured so that $\phi=0$ at a perihelion. This requires that $B=0$ (because $\left.\frac{d s}{d \phi}\right|_{\phi=0}=0$ at a perihelion). Thus, we may rewrite the solution as

$$
s=\frac{1}{r}=A \cos (\lambda \phi)+D / \lambda^{2} .
$$

In the nonrelativistic case $A / D$ is just the eccentricity of the Kepler ellipse, which can be randomly chosen within the interval $[0,1]$. However, in the relativistic case we must substitute the solution in Eq. (6) into the constant-energy equation in order to determine $A$. Namely, we insert Eq. (6) into the equation

$$
E_{0}=c^{2} \sqrt{1+p_{r}^{2} / c^{2}+L^{2} / r^{2} c^{2}}-Z / r
$$

and note that at a perihelion $\phi=0$ and $p_{r}=0$. One obtains thereby the following result for the constant $A$ :

$$
A=\frac{\sqrt{c^{2} L^{2} E_{0}^{2}-c^{4}\left(c^{2} L^{2}-Z^{2}\right)}}{c^{2} L^{2}-Z^{2}} .
$$

In this way, we obtain the corresponding result for the relativistic Kepler orbit solution:

$$
\begin{aligned}
s=\frac{1}{r}= & \frac{\sqrt{c^{2} L^{2} E_{0}^{2}-c^{4}\left(c^{2} L^{2}-Z^{2}\right)}}{c^{2} L^{2}-Z^{2}} \cos \left(\sqrt{1-Z^{2} / c^{2} L^{2}} \phi\right) \\
& +\frac{Z E_{0}}{c^{2} L^{2}-Z^{2}} .
\end{aligned}
$$

In order that the orbit is stable, we must enforce the following conditions: (1) $1-Z^{2} / c^{2} L^{2}>0$ and (2) $c^{2} L^{2} E_{0}^{2}-c^{4}\left(c^{2} L^{2}\right.$
$\left.-Z^{2}\right)>0$. These conditions serve to confine the possible values of the magnitude of the angular momentum-i.e., $Z / c$ $<|L|<Z / \sqrt{c^{2}-E_{0}^{2} / c^{2}}$. Within this range, we may randomly choose (positive and negative) values for the angular momentum $L$ and then use the orbit equation (9) to sample points $\left(x_{0}, y_{0}\right)=(r \cos (\phi), r \sin (\phi))$ along the corresponding relativistic Kepler ellipse as functions of the polar angle $\phi$. Note that the precession of the relativistic Kepler ellipse is essentially included in the orbit equation (9) in which the perihelion shift per period equals $\delta \phi=\left(\sqrt{\frac{c^{2} L^{2}}{c^{2} L^{2}-Z^{2}}}-1\right) 2 \pi$. After obtaining the set of trajectory locations $\left(x_{0}, y_{0}\right)$, we can determine the corresponding momenta $\left(p_{x_{0}}, p_{y_{0}}\right)$ by solving the equations for the total energy $E_{0}$ and the angular momentum $L$ :

$$
\begin{gathered}
E_{0}=\gamma_{0} c^{2}-Z / \sqrt{x_{0}^{2}+y_{0}^{2}}, \\
L=x_{0} p_{y_{0}}-y_{0} p_{x_{0}},
\end{gathered}
$$

where $\gamma_{0}=\sqrt{1+\left(p_{x_{0}}^{2}+p_{y_{0}}^{2}\right) / c^{2}}$. Repeating the above procedure, one obtains a set of points $\left(x_{0}, p_{x_{0}}, y_{0}, p_{y_{0}}\right)$ that are randomly distributed along the relativistic Kepler ellipses corresponding to our randomly chosen (positive and negative) values of angular momenta, $L$.

\section{Second step: Rotation of the planar ensemble}

Choosing arbitrary sets of Euler angles $(\theta, \varphi, \eta)$, we next rotate a given relativistic Kepler orbit into three dimensions, as follows [68]:

$$
\left(\begin{array}{c}
x_{0}^{\prime} \\
y_{0}^{\prime} \\
z_{0}^{\prime}
\end{array}\right)=R\left(\begin{array}{c}
x_{0} \\
y_{0} \\
0
\end{array}\right), \quad\left(\begin{array}{c}
p_{x_{0}}^{\prime} \\
p_{y_{0}}^{\prime} \\
p_{z_{0}}^{\prime}
\end{array}\right)=R\left(\begin{array}{c}
p_{x_{0}} \\
p_{y_{0}} \\
0
\end{array}\right),
$$

where the rotation matrix is expressed as

$$
R=\left(\begin{array}{ccc}
-\sin \varphi \sin \eta+\cos \varphi \cos \theta \cos \eta & -\sin \varphi \cos \eta-\cos \varphi \cos \theta \sin \eta & \cos \varphi \sin \theta \\
\cos \varphi \sin \eta+\sin \varphi \cos \theta \cos \eta & \cos \varphi \cos \eta-\sin \varphi \cos \theta \sin \eta & \sin \varphi \sin \theta \\
-\sin \theta \cos \eta & \sin \theta \sin \eta & \cos \theta
\end{array}\right)
$$

\section{Tests for validity of the microcanonical ensemble and of the Monte Carlo method}

The radial and momentum distributions of the classical microcanonical ensemble constructed as described above can be tested by comparison with the known quantum- mechanical ground-state radial and momentum probability distributions for a hydrogenic ion. We have verified that the ensemble described above truly mimics the quantummechanical ground state of interest. Such a "phase-spaceaveraging" method [61] has been used extensively to investigate atomic collisions $[69,70]$, Rydberg atom ionization by 
TABLE I. Comparison of ADK tunneling ionization and classical trajectory Monte Carlo (CTMC) ionization probabilities for different HCI species and peak laser intensities. The laser wavelength is $\lambda=1054 \mathrm{~nm}$, and the 15 -cycle trapezoidal laser pulse is assumed to have a 5-cycle linear turn-on ("ramp") and turn-off and a 5-cycle flat top.

\begin{tabular}{rrrrrr}
\hline \hline HCI species & Intensity $\left(\mathrm{W} / \mathrm{cm}^{2}\right)$ & ADK (ramp) & CTMC (ramp) & ADK (flat top) & CTMC (flat top) \\
\hline $\mathrm{Fe}^{25+}(Z=26)$ & $2.000 \times 10^{22}$ & $0.259 \%$ & $0.187 \%$ & $26.206 \%$ & $53.243 \%$ \\
$\mathrm{Co}^{26+}(Z=27)$ & $2.489 \times 10^{22}$ & $0.261 \%$ & $0.228 \%$ & $26.665 \%$ & $57.670 \%$ \\
\hline
\end{tabular}

microwaves [61], and numerous intense laser-atom processes [9].

For the problem considered here, the major reason for using the classical relativistic Monte Carlo method is that a quantum-mechanical calculation is intractable, even in two dimensions, owing to the large laser-induced excursions made by the ionized electron. The major quantummechanical effect that is neglected is quantum-mechanical tunneling ionization of the bound electron. We have verified, however, that for the highly charged ions considered here quantum-mechanical tunneling ionization is negligible over the five-cycle rise time of the laser pulses considered. Indeed, the tunneling probability decreases exponentially with an argument proportional to $-Z^{3}$, where $Z$ is the ionic charge [71]. (Note that tunneling rates are small because the laser electric fields, despite their large absolute values, are nevertheless small compared to the Coulomb fields experienced by the hydrogenic electrons in the highly charged ions considered here.) As an example, using Ammosov-Delone-Krainov (ADK) tunneling ionization rates [71] we estimate that during the five-cycle linear rise time of a laser pulse having a peak intensity of $I=2 \times 10^{22} \mathrm{~W} / \mathrm{cm}^{2}$ the ionization probability for a hydrogenic ion having $Z=26$ is less than $0.3 \%$. Furthermore, any tunneling that occurs during the subsequent five cycles that the laser pulse is at its peak amplitude would only enhance our predicted results.

We emphasize that in using a classical, relativistic approach we expect our predictions for the probability of electron ionization to be only approximately correct. However, accurate predictions for electron ionization probabilities are unnecessary for our purposes in this paper, which are to analyze the energy and angular distributions of those electrons that are ionized by a short laser pulse. These distributions are independent of the absolute magnitude of the ionization probability, which requires in principle a quantummechanical calculation. In fact, however, our classical estimates of the probability of ionization (defined as the fraction of classical electron trajectories that result in ionization) are remarkably close to our quantum estimates of the ionization probabilities. As discussed in Sec. III C below (cf. Table I), the classical estimates for ionization during the five-cycle ramp of the leading edge of the laser pulse agree to within $30 \%$ (or better) with the quantum estimates and on the fivecycle flat top of the laser pulse differ from the quantum estimates by only a factor of 2 . The key point is that our model provides the correct order of magnitude of the electron ionization probability during each phase of the laser pulse. For those electron trajectories that result in ionization, our classical relativistic description of the post-ionization acceleration of the electrons in the field of the laser pulse is expected to provide an accurate account of the energy and angular distributions. This is expected because these distributions are insensitive to the mechanism by which electrons are ionized, provided only that the electrons are ionized primarily at the peak amplitude of the laser pulse.

Typically we employ a microcanonical ensemble comprising a total of 500000 points in phase space. We then randomly choose a starting point $\left(x_{0}^{\prime}, p_{x_{0}}^{\prime}, y_{0}^{\prime}, p_{y_{0}}^{\prime}, z_{0}^{\prime}, p_{z_{0}}^{\prime}\right)$ from this ensemble as the initial condition for a particular trajectory and then integrate the above classical equations (2) in the presence of both the Coulomb field and the laser field. We continue the integration until such time that the laser pulse bypasses the electron (i.e., the laser field experienced by the electron becomes zero). In this way, we obtain results for one individual trajectory. We then repeat the same process until we obtain statistically unchanged results-i.e., until the normalized energy and angular distributions remain essentially unchanged.

\section{Tight-focusing effects}

It is well known that the field components of a propagating laser beam must satisfy Maxwell's equations. When focused by a lens or by a reflecting mirror, a laser beam in the region of the focus is generally well described by a Gaussian profile function provided the beam waist $w_{0}$ is much greater than the laser wavelength $\lambda$. However, if a laser beam is so tightly focused that the beam waist is of the order of the laser wavelength, then a Gaussian beam description becomes inaccurate. Specifically, tight focusing of a laser beam results in non-Gaussian field components in all three dimensions, even for a laser beam that is linearly polarized outside the focal region. This effect has been analyzed in a number of references [37,49-52]. Thus, we give in what follows only a brief overview of the theory underlying our calculations of these effects.

A monochromatic laser beam propagating in vacuum may be described by means of a vector potential $\mathbf{A}$ that satisfies the Helmholtz equation $\nabla^{2} \mathbf{A}+k^{2} \mathbf{A}=0$, where $k=\omega / c$ is the wave number, $\omega$ is the laser frequency, and $c$ is the speed of light. In the Lorentz gauge $\left(\boldsymbol{\nabla} \cdot \mathbf{A}+\frac{1}{c} \frac{\partial \Phi}{\partial t}=0\right)$, the scalar potential $\Phi$ can be written in terms of the vector potential-i.e., $\Phi=(i / k) \boldsymbol{\nabla} \cdot \mathbf{A}$-as can the electric and magnetic field vectors:

$$
\mathbf{E}=-(i / k) \boldsymbol{\nabla}(\boldsymbol{\nabla} \cdot \mathbf{A})-i k \mathbf{A}, \quad \mathbf{B}=\boldsymbol{\nabla} \times \mathbf{A} .
$$

For a laser beam assumed to be propagating along the $+z$ axis and linearly polarized along the $x$ axis, the timeindependent part of the vector potential is given by $\mathbf{A}$ $=f(x, y, z) e^{-i k z} \mathbf{e}_{x}$, where $\mathbf{e}_{x}$ is the unit vector along the $x$ axis. 
Making a change of spatial variables-e.g., $\alpha=x / w_{0}, \beta$ $=y / w_{0}$, and $\eta=z /\left(k w_{0}^{2}\right)$-we obtain from the Helmholtz equation satisfied by the vector potential $\mathbf{A}$ the following equation for the function $f(x, y, z)$ :

$$
\left(\frac{\partial^{2}}{\partial \alpha^{2}}+\frac{\partial^{2}}{\partial \beta^{2}}-2 i \frac{\partial}{\partial \eta}\right) f(x, y, z)=-s^{2} \frac{\partial^{2} f(x, y, z)}{\partial \eta^{2}},
$$

where the parameter $s=1 / k w_{0}=\frac{1}{2 \pi} \frac{\lambda}{w_{0}}$ is a small number for $\lambda \ll w_{0}$. We now formally expand the solution in powers of the parameter $s$ :

$$
f(\alpha, \beta, \eta)=f_{0}(\alpha, \beta, \eta)+s^{2} f_{2}(\alpha, \beta, \eta)+s^{4} f_{4}(\alpha, \beta, \eta)+\cdots .
$$

Substituting this expansion into Eq. (14) and equating terms of the same order in the parameter $s$, we obtain three equations for the functions $f_{0}, f_{2}$, and $f_{4}$ in Eq. (15). The zerothorder solution is $f_{0}=i Q \exp \left(-i \rho^{2} Q\right)$, where $Q=1 /(i+2 \eta)$ and $\rho^{2}=\alpha^{2}+\beta^{2}$; it is the familiar Gaussian beam profile. The higher-order solutions are given by $f_{2}=\left(2 i Q+i \rho^{4} Q^{3}\right) f_{0}$ and $f_{4}=\left(-6 Q^{2}-3 \rho^{4} Q^{4}-2 i \rho^{6} Q^{5}-0.5 \rho^{8} Q^{6}\right) f_{0}$. Finally, we obtain the vector potential, $\mathbf{A}(x, y, z)=\left[f_{0}(x, y, z)+s^{2} f_{2}(x, y, z)\right.$ $\left.+s^{4} f_{4}(x, y, z)\right] e^{i(\omega t-k z)} \mathbf{e}_{x}$, which satisfies the Helmhotz equation up to fifth-order terms in the parameter $s$. The resulting electric and magnetic field components are derived from Eq. (13):

$$
\begin{aligned}
& E_{x}=E_{0}\left\{1+s^{2}\left[-\rho^{2} Q^{2}+i \rho^{4} Q^{3}-2 Q^{2}\left(x / w_{0}\right)^{2}\right]\right. \\
& +s^{4} \times\left[2 \rho^{4} Q^{4}-3 i \rho^{6} Q^{5}-0.5 \rho^{8} Q^{6}+\left(x / w_{0}\right)^{2}\right. \\
& \left.\left.\times\left(8 \rho^{2} Q^{4}-2 i \rho^{4} Q^{5}\right)\right]\right\} i Q e^{-i \rho^{2} Q} e^{i \omega t-i k z}, \\
& E_{y}=E_{0}\left\{s^{2}\left(-2 Q^{2} x y / w_{0}^{2}\right)+s^{4}\left(8 \rho^{2} Q^{4}-2 i \rho^{4} Q^{5}\right)\right. \\
& \left.\times x y / w_{0}^{2}\right\} i Q e^{-i \rho^{2} Q} e^{i \omega t-i k z}, \\
& E_{z}=E_{0}\left\{s\left(-2 Q x / w_{0}\right)+s^{3}\left(6 \rho^{2} Q^{3}-2 i \rho^{4} Q^{4}\right) x / w_{0}\right. \\
& \left.+s^{5}\left(-20 \rho^{4} Q^{5}+10 i \rho^{6} Q^{6}+\rho^{8} Q^{7}\right) x / w_{0}\right\} \\
& \times i Q e^{-i \rho^{2} Q} e^{i \omega t-i k z} \text {, } \\
& B_{x}=E_{0}\left\{s^{2}\left(-2 Q^{2} x y / w_{0}^{2}\right)+s^{4}\left(8 \rho^{2} Q^{4}-2 i \rho^{4} Q^{5}\right)\right. \\
& \left.\times x y / w_{0}^{2}\right\} i Q e^{-i \rho^{2} Q} e^{i \omega t-i k z}, \\
& B_{y}=E_{0}\left\{1+s^{2}\left(-\rho^{2} Q^{2}+i \rho^{4} Q^{3}-2 Q^{2} y^{2} / w_{0}^{2}\right)\right. \\
& +s^{4}\left[2 \rho^{4} Q^{4}-3 i \rho^{6} Q^{5}-0.5 \rho^{8} Q^{6}+\left(y / w_{0}\right)^{2}\right. \\
& \left.\left.\times\left(8 \rho^{2} Q^{4}-2 i \rho^{4} Q^{5}\right)\right]\right\} i Q e^{-i \rho^{2} Q} e^{i \omega t-i k z}, \\
& B_{z}=E_{0}\left\{s\left(-2 Q y / w_{0}\right)+s^{3}\left(6 \rho^{2} Q^{3}-2 i \rho^{4} Q^{4}\right) y / w_{0}\right. \\
& \left.+s^{5}\left(-20 \rho^{4} Q^{5}+10 i \rho^{6} Q^{6}+\rho^{8} Q^{7}\right) y / w_{0}\right\} \\
& \times i Q e^{-i \rho^{2} Q} e^{i \omega t-i k z} \text {, }
\end{aligned}
$$

where $E_{0}$ is the peak field amplitude at the center of the laser focus (i.e., $x=y=z=0$ ), and where the parameters $\rho^{2}$ and $Q$ may be rewritten as $\rho^{2}=\left(x^{2}+y^{2}\right) / w_{0}^{2}$ and $Q=b /(i b+2 z)$, where $b$ is the focal length, $b=2 \pi w_{0}^{2} / \lambda$. From the above
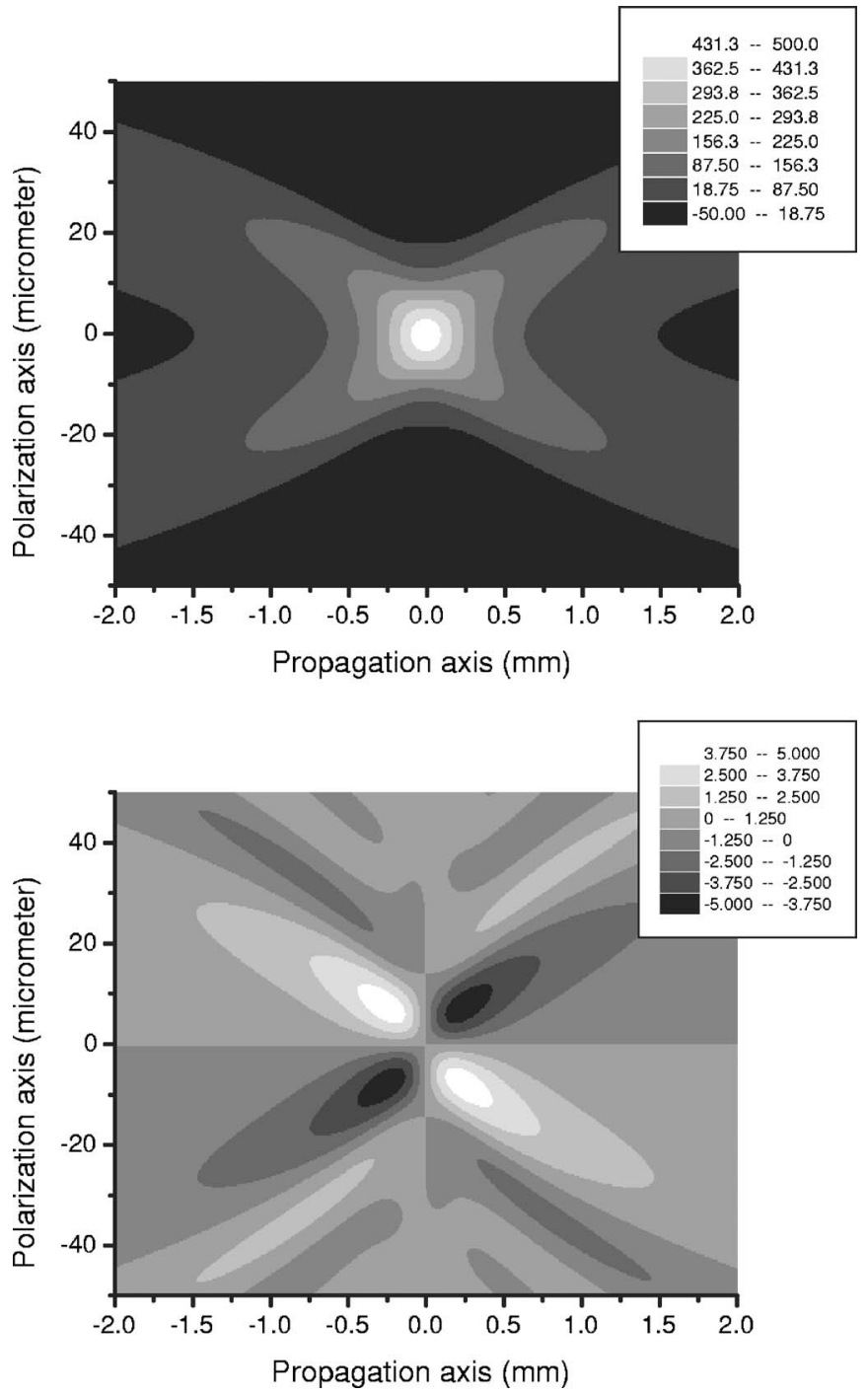

FIG. 2. The spatial profiles of the real part of the laser electric field components $E_{x}(x, y=0, z)$ (upper panel) and $E_{z}(x, y=0, z)$ (lower panel) (with field strengths given in atomic units). The laser beam waist is $w_{0}=10 \mu \mathrm{m}$ and the peak laser intensity in the focal region is $8 \times 10^{21} \mathrm{~W} / \mathrm{cm}^{2}$. The longitudinal field component $E_{z}(x, y=0, z)$ is smaller than the main component $E_{x}(x, y=0, z)$ by two orders of magnitude; unlike $E_{x}, E_{z}$ is asymmetric with respect to the laser focus.

equations, we see that for a linearly polarized laser pulse the largest electric and magnetic field components are $E_{x}$ and $B_{y}$, both of which have leading terms of order $s^{0}$. The other field components arise because of the tight focusing and have magnitudes proportional to low powers of the parameter $s$ $=\frac{1}{2 \pi} \frac{\lambda}{w_{0}}$. Thus, if the beam waist $w_{0}$ is much larger than the laser wavelength, these field components can be ignored. However, if the beam waist approaches the order of magnitude of the laser wavelength (as in the case of tight focusing), these focusing-induced fields become significant. In Fig. 2 we show how the longitudinal field component $E_{z}$ (lower panel) compares to the main electric field component $E_{x}$ (upper panel). In the case that $w_{0}=10 \mu \mathrm{m}$, the longitudinal field $E_{z}$ is much smaller than $E_{x}$ (by approximately two orders of 
magnitude). Figure 2 also indicates that the leading longitudinal field component $E_{z}$ is asymmetric with respect to the center of the focus. Although this component is very small, it has significant effects on the ionized electron angular and energy distributions, as shown in the next section.

\section{RESULTS AND DISCUSSION}

Using the three-dimensional (3D) Monte Carlo simulation method described above, we have investigated the acceleration of electrons by an ultrashort, superstrong laser pulse. We treat the electron acceleration in vacuum, in which the electrons are either free electrons or those stripped from highly charged ions by the laser pulse. Typically, we have calculated from 4000 to 12000 electron trajectories for each case in order to achieve statistically unchanged results.

\section{A. Free electrons as targets}

In our 3D Monte Carlo simulations, the laser wavelength is equal to $\lambda=1054 \mathrm{~nm}$ (as for the hybrid Ti:sapphire$\mathrm{Nd}$ :glass laser system [2]) and the laser peak intensity is assumed to be $2 \times 10^{22} \mathrm{~W} / \mathrm{cm}^{2}$ when focused to a spot having a beam waist $w_{0}=10 \mu \mathrm{m}$. These laser parameters are within current or near-future experimental capabilities $[2,6]$. The laser pulse is assumed to be linearly polarized along the $x$ axis and to propagate along the $z$ axis. It comprises 15 laser cycles (with a linear turn-on and -off of five-cycles and a five-cycle flat top). At first glance it may seem that with such an ultraintense laser pulse, the production of ultraenergetic $\mathrm{GeV}$ electrons is not surprising. However, it is easy to show that achieving $\mathrm{GeV}$ energies solely with such an intense laser pulse is not possible. To elucidate this, we have carried out Monte Carlo simulations using free electrons as targets-i.e., in which free electrons are assumed to be initially at rest and randomly distributed within a cylindrical volume oriented along the $z$ axis, centered about the origin, and having a radius of $5 \mu \mathrm{m}$ and a length of $1 \mathrm{~mm}$. In Fig. 3(a) we plot the final electron energies as a function of their ejection angles $\theta$. We find that electrons located initially before the focus (i.e., located in the half of the cylindrical region defined by $z<0$ ) have a different final-state angular and energy distribution than those electrons located initially after the focus (i.e., in the cylindrical region defined by $z>0$ ). Specifically, the electrons located initially in the $z<0$ region are accelerated to lower final-state energies and larger ejection angles [indicated by the dashed circle in Fig. 3(a)]. Figure 3(b) shows the normalized energy distribution for the 12000 electron trajectories that were calculated. It indicates that most electrons acquire energies of less than $200 \mathrm{MeV}$, even though they interact with such a superstrong laser pulse.

To illustrate the difficulties of using free electrons as targets for laser acceleration of electrons to $\mathrm{GeV}$ energies, we present results for a single trajectory in order to exhibit how a free electron responds to the superintense laser field inside the region of the laser focus. Figure 4 presents the laser electric field components experienced by the electron in its rest frame, $E_{x}(\rho, z, t)$ and $E_{z}(\rho, z, t)$, which are plotted versus the interaction time in the laboratory frame. One observes an
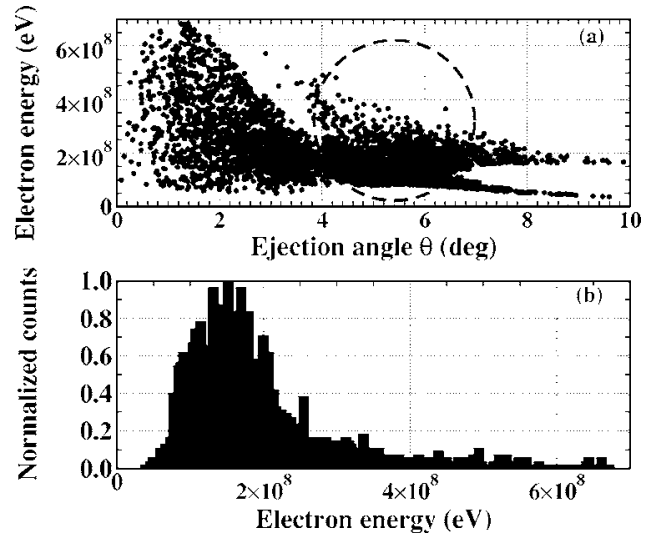

FIG. 3. Monte Carlo simulation results for free electrons interacting with a superstrong laser pulse. (a) The resulting electron energies are plotted as a function of their ejection angles $\theta$. (b) The normalized distribution of electron energies, which shows that most electrons acquire energies of less than $200 \mathrm{MeV}$. The laser pulse has a peak intensity of $2 \times 10^{22} \mathrm{~W} / \mathrm{cm}^{2}$, a wavelength of $1054 \mathrm{~nm}$, a beam waist in the focal region of $w_{0}=10 \mu \mathrm{m}$, and a duration of 15 laser cycles, including a five-cycle linear turn-on and -off and a five-cycle flat top. The free electrons are assumed to be at rest initially and to be distributed randomly within a cylindrical volume (having a radius of $5 \mu \mathrm{m}$ and a length of $1 \mathrm{~mm}$ ) that is oriented along the $z$ axis and centered about the origin.

enormous relativistic Doppler effect; i.e., during the course of about 1000 laser periods in the laboratory frame, the electron experiences only about two laser cycles in its rest frame before it is expelled from the region of the laser focus and no longer experiences any electric fields in its rest frame. The laser cycles that the electron therefore "sees" are only those few oscillations occurring during the laser turn-on stage, which have electric field amplitudes that are significantly smaller than the peak laser electric field amplitude of $\sim 800$ a.u. (i.e., $\sim 4 \times 10^{12} \mathrm{~V} / \mathrm{cm}$ ) Furthermore, as the electron moves out of the region of the laser focus, it experiences also the longitudinal field component $E_{z}(\rho, z, t)$, which either accelerates or decelerates the electron, depending on its phase. The electron's main momentum components and en-

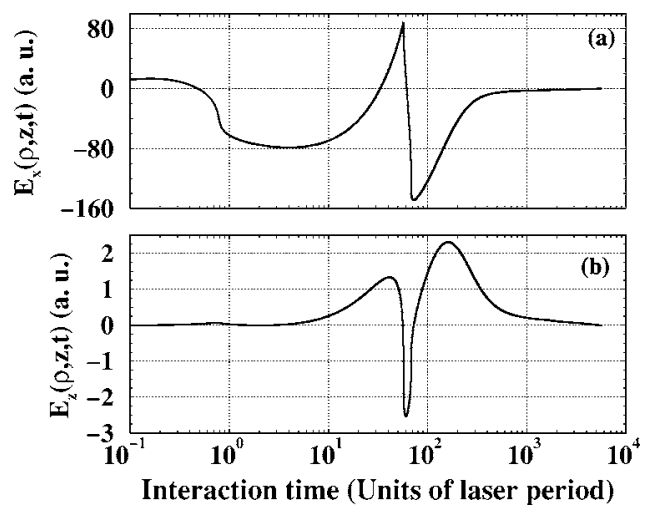

FIG. 4. Laser electric field components in the rest frame of a free electron interacting with a superintense laser field, plotted vs interaction time in the laboratory frame: (a) $E_{x}(\rho, z, t)$ and (b) $E_{z}(\rho, z, t)$. The laser parameters are the same as those in Fig. 3. 


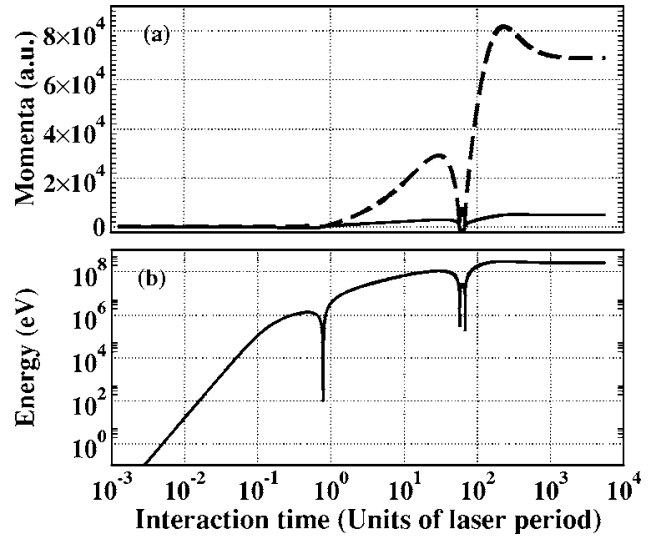

FIG. 5. The momentum components and energy for the same electron trajectory considered in Fig. 4, plotted as functions of the electron-laser interaction time in the laboratory frame: (a) electron momentum components $p_{z}$ (dashed line) along the laser propagation axis and $p_{x}$ (solid line) along the direction of laser polarization and (b) electron energy.

ergy in the laboratory frame are plotted in Fig. 5 for the same trajectory considered in Fig. 4. One sees that for such a superintense laser field, the electron momentum component along the laser propagation direction $p_{z}$ quickly becomes much larger than its momentum component along the direction of laser polarization $p_{x}$ owing to the Lorentz force $\mathbf{v}$ $\times \mathbf{B}$. Nevertheless, because the electron on the trajectory considered is expelled from the region of the laser focus after only two laser cycles (in its rest frame), its final kinetic energy is only approximately $250 \mathrm{MeV}$. Achieving efficient electron acceleration with free electrons thus appears to require their injection into the region of the laser focus $[44,45]$. However, in order to overcome the ponderomotive potential, the injected electrons must have initial kinetic energies of $\sim 10 \mathrm{MeV}$, which thus requires a separate acceleration step. Moreover, the angle at which the electrons are injected into the focal region is important. An alternative way to accelerate electrons to $\mathrm{GeV}$ energies using superintense laser electric fields is to employ highly charged ions as targets [42], which we discuss next.

\section{B. Highly charged ions as targets}

The key idea for using highly charged ions (HCIs) as electron sources for laser acceleration is that the deep atomic potential that binds the electron in a highly charged ion can prevent its ionization until the laser pulse reaches its maximum intensity, so that the ionized electron will experienceand be accelerated by-the maximum value of the laser electric field before its eventual expulsion from the region of the laser focus. This scenario implies that one matches the ion target to the intensity of the laser field so that little ionization occurs during the rise time of the laser pulse, but significant ionization occurs in the vicinity of its maximum intensity. Since nowadays essentially any charge state of any atom can be produced [72], there is great flexibility in matching a particular laser peak intensity (which can be varied by adjusting the beam waist in the focal region) to a particular highly

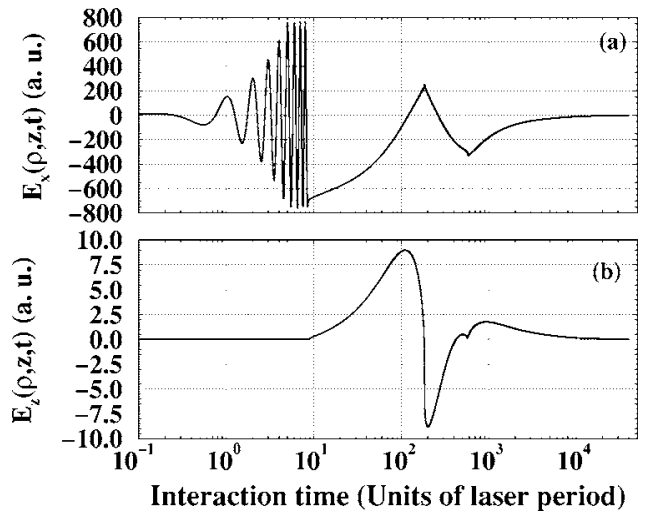

FIG. 6. Laser electric field components in the rest frame of an electron initially bound in the highly charged $\mathrm{Fe}^{25+}$ ion and ionized after 8.5 laser periods, plotted vs the interaction time in the laboratory frame (measured in units of the laser period). (a) $E_{x}(\rho, z, t)$, the transverse field along the laser polarization direction. (b) $E_{z}(\rho, z, t)$, the longitudinal field along the direction of laser propagation. The laser parameters are the same as those in Fig. 3. For this particular electron trajectory, the ion is located initially at the center of the laser focus-i.e., at $(x=0, y=0, z=0)$.

charged ion. In the calculations we present here we have therefore chosen highly charged, hydrogenic ions that match well with the parameters of the laser pulse we consider without regard to any other considerations.

To illustrate the difference between using a highly charged ion as a target and using free electrons as targets, consider a particular trajectory for an electron ionized from a hydrogenlike $\mathrm{Fe}^{25+}$ ion by an ultraintense laser pulse having the same parameters as for the free electron case considered above. Figure 6 presents the laser electric field components experienced by the electron in its rest frame, $E_{x}$ and $E_{z}$, which are plotted versus the interaction time in the laboratory frame. The $\mathrm{Fe}^{25+}$ ion in this example is placed initially at the center of the laser focus. From Fig. 6 one sees that owing to the tight binding provided by the nuclear Coulomb field, the electron remains bound during the turn-on (i.e., the first five cycles) of the laser pulse. It remains bound during an additional three (peak-amplitude) cycles of the laser pulse before it becomes ionized. Before ionization, Fig. 6 shows that the number of laser cycles experienced by the electron coincides with the number of laser periods in the laboratory frame. Following ionization, however, the electron on this particular trajectory is quickly accelerated to velocities close to the speed of light. It experiences somewhat more than one laser cycle in its rest frame during a time in the laboratory of about 1000 laser periods, which is a consequence of the relativistic Doppler shift of the laser field in the rest frame of the electron. The electron therefore experiences the peak value of the laser electric field (in the direction of laser polarization) over an extended time in the laboratory frame; i.e., it "rides" or "surfs" on one of the peak (laser amplitude) waves within the laser pulse. Eventually, however, as shown in Fig. 6 , the laser electric field along the polarization direction changes sign in the electron rest frame (because the electron velocity $v_{z}$ remains slightly less than the speed of light, $c$, resulting in phase slippage). As shown in Fig. 6(b), this 


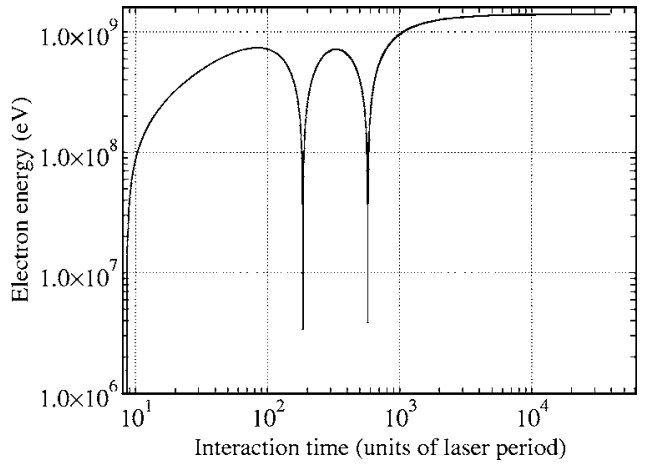

FIG. 7. Ionized electron energy plotted vs the interaction time in the laboratory frame for the specific electron trajectory shown in Fig. 6 for an electron ionized from $\mathrm{Fe}^{25+}$.

phase slippage is furthered by the longitudinal electric field $E_{z}$ in the laser focal region, which for this particular electron trajectory provides a slight deceleration of electron motion along the $z$ axis. In fact, the laser field changes sign twice along this particular trajectory. Interestingly, we see that the peak positive value of $E_{x}$ at around the time $190 \mathrm{~T}$ has a significantly smaller magnitude than at the peak negative value of $E_{x}$ at close to $600 \mathrm{~T}$, indicating that during these times the electron on this trajectory gets closer to the laser propagation axis as it is accelerated along positive $z$ and therefore experiences a larger (negative) field at the later time owing to the spatial distribution of our laser pulse field [cf. Eq. (2) of Ref. [42]].

The ionized electron's energy is plotted versus the interaction time in the laboratory frame in Fig. 7. The ionized electron's momentum components along the laser propagation axis $(z$ axis $)$ and along the laser polarization direction ( $x$ axis) are shown in Fig. 8. One sees that the electron quickly gains and loses energy as the laser electric field in its rest frame changes sign. During the third half laser cycle following its ionization, however, the electron gains a large amount of energy and consequently leaves the laser focus with a final energy of nearly $1.4 \mathrm{GeV}$. Note that (as shown in Fig. 8) the electron propagates continuously along the $z$ axis

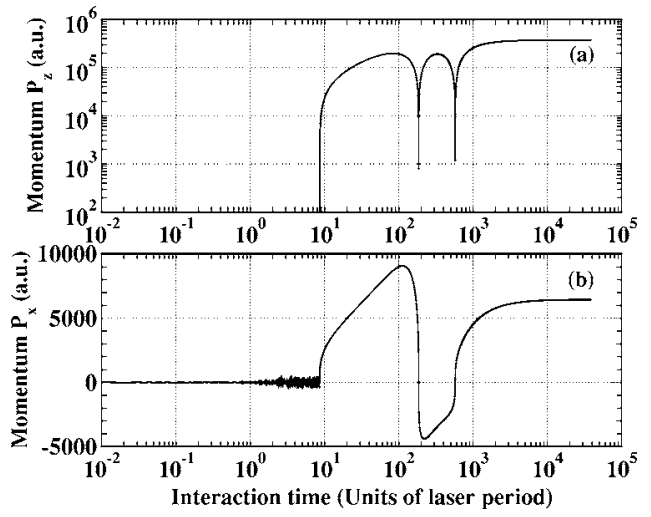

FIG. 8. Ionized electron momentum components plotted vs the interaction time in the laboratory frame for the specific electron trajectory shown in Fig. 6 for an electron ionized from $\mathrm{Fe}^{25+}$. (a) Momentum along the laser propagation axis ( $z$ axis). (b) Momentum along the laser polarization axis ( $x$ axis).

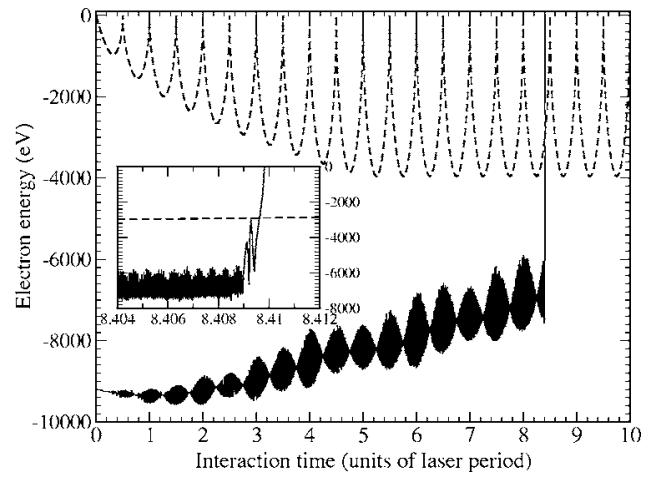

FIG. 9. The process of classical electron escape over the effective potential barrier that is formed by the laser field and the Coulomb field of the nucleus. The total electron energy in an $\mathrm{Fe}^{25+}$ ion is plotted as a function of the interaction time (measured in units of the laser period) for the specific trajectory considered in Fig. 6. The dashed line is the energy value of the effective potential barrier, which oscillates with the laser field. The inset shows the instant of electron escape over the potential barrier (i.e., electron ionization) on an expanded time scale.

despite the change in sign of $E_{x}$ between about $80 \mathrm{~T}$ and $300 \mathrm{~T}$. Even though the electron is decelerated during this time, its velocity along the $z$ axis remains close to that of the speed of light, $c$, with its minimum energies at around $190 \mathrm{~T}$ and $600 \mathrm{~T}$ still in the range of 3-4 MeV. In part, this uninterrupted motion along the positive $z$ axis is aided by the change in sign of $E_{z}$ between around $190 \mathrm{~T}$ and $400 \mathrm{~T}$. The net result is that along this trajectory the electron keeps moving with the laser pulse until it is eventually out of the region of the laser focus.

The total energy of the electron prior to and just after its ionization by the laser pulse is shown in Fig. 9 for the same trajectory as in Figs. 6 and 7. The dashed line in the upper part of the figure (as well as in the inset) shows the energy value of the effective potential barrier-i.e., the sum of the interaction energies of the electron with the laser electric field and with the Coulomb field of the nucleus. One sees that, as expected, this effective potential barrier takes a minimum value twice per laser cycle after the laser pulse has reached its maximum amplitude-i.e., at $t=5 T, 5.5 T, 6 T, \ldots$, where $T$ is the laser period. One sees from Fig. 9 that the electron gains energy gradually from the laser pulse. During the five cycles of the laser pulse turn-on, the electron's total energy remains negative (i.e., it remains bound), indicating that the electron truly does survive the rising front edge of the laser pulse. The inset figure in Fig. 9 indicates on an expanded time scale the instant at which the electron is ionized-i.e., gains sufficient energy from the laser pulse to leap above its effective potential barrier and escape so that it can be accelerated by the peak field of the laser pulse, as shown in Figs. 6 and 7.

Finally, we note that for the electron trajectory considered in Figs. 6-9, which we regard as a typical ionized electron trajectory, the radiative losses during electron acceleration are estimated to be very small. Our estimate employed the well-known relativistic generalization of Lamor's formula for the instantaneous radiated power [73], 


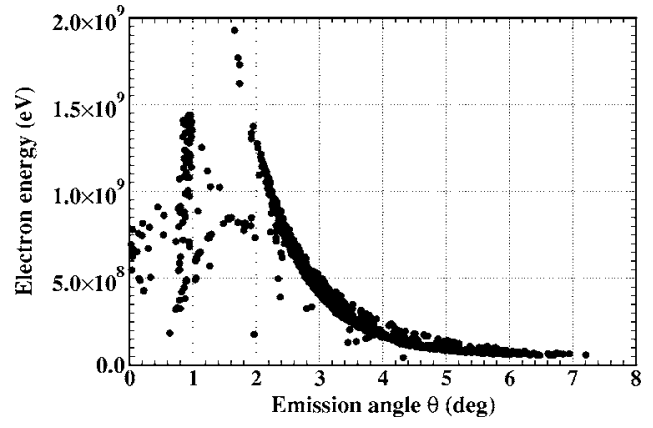

FIG. 10. Monte Carlo results for highly charged $\mathrm{Fe}^{25+}$ ions interacting with an intense laser pulse having the same parameters as described in Fig. 3, including a beam waist equal to $w_{0}=10 \mu \mathrm{m}$. Ionized electron energies are plotted vs their ejection angle $\theta$ with respect to the laser propagation axis. The ions are assumed to be placed at the center of the laser focus.

$$
P=\frac{2 \gamma^{6}}{3 c}\left[\left(\frac{d \beta}{d t}\right)^{2}-\left(\beta \times \frac{d \beta}{d t}\right)^{2}\right]
$$

where $c=137.036$ is the speed of light in atomic units, $\beta$ $=\mathbf{v} / c$ is the electron's relativistic velocity divided by $c$, and $\gamma$ is the usual relativistic factor. For the ionized electron trajectory considered in Figs. 6-9 we calculated the instantaneous radiated power and integrated it over time in order to obtain an estimate for the total radiative energy loss. For this trajectory, we obtained a total energy loss of only $\approx 14.2 \mathrm{keV}$, which is a factor of $\approx 10^{-5}$ times smaller than the total energy gain of $1.4 \mathrm{GeV}$ along this trajectory. For this reason, we did not consider radiative damping in our calculations.

Using highly charged $\mathrm{Fe}^{25+}$ ions as targets, we have carried out simulations of ultraintense laser acceleration of electrons. Monte Carlo results for the case in which the ions are placed at the center of the laser focus are shown in Fig. 10, in which the ionized electron energies are plotted versus their ejection angles $\theta\left[\right.$ defined as $\left.\theta=\cos ^{-1}\left(p_{z} / \sqrt{p_{x}^{2}+p_{y}^{2}+p_{z}^{2}}\right)\right]$. The laser parameters are the same as those considered in Fig. 3; in particular, the laser beam waist is $w_{0}=10 \mu \mathrm{m}$. One sees that, in general, ionized electrons having small ejection angles have higher energies. However, the relation between electron ejection angle and final electron kinetic energy is not monotonic, owing to the longitudinal laser field component $E_{z}$. In fact, for electrons having ejection angles $\theta<2^{\circ}$, for each electron ejection angle there is typically a widespread distribution of electron kinetic energies. Nevertheless, one sees that there are many trajectories for electrons ionized from the highly charged $\mathrm{Fe}^{25+}$ ion that have final electron energies above $1 \mathrm{GeV}$, which contrasts significantly with the case considered earlier of using the same ultraintense laser pulse to accelerate free electrons [cf. Fig. 3(a)].

Figure 11 presents Monte Carlo results for the case that the laser beam waist in the focal region is increased to $w_{0}$ $=20 \mu \mathrm{m}$ so as to decrease the laser's longitudinal field component $E_{z}$. The laser peak intensity $\left(2 \times 10^{22} \mathrm{~W} / \mathrm{cm}^{2}\right)$, wavelength $(\lambda=1054 \mathrm{~nm})$, and pulse duration (15 laser cycles) are the same as those employed in Figs. 3 and 10. Compared to the results shown in Fig. 10 for a laser beam waist of $w_{0}$

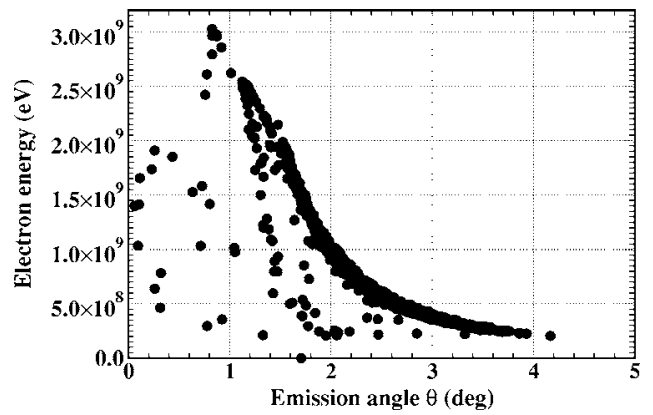

FIG. 11. Monte Carlo results for highly charged $\mathrm{Fe}^{25+}$ ions interacting with an intense laser pulse having the same parameters as described in Fig. 3 except that the beam waist here is equal to $w_{0}$ $=20 \mu \mathrm{m}$. Ionized electron energies are plotted vs their ejection angle $\theta$ with respect to the laser propagation axis. The ions are assumed to be placed at the center of the laser focus.

$=10 \mu \mathrm{m}$, the number of trajectories shown in Fig. 11 for small ejection angles that have final electron energies greater than $1 \mathrm{GeV}$ is far greater. In fact, nearly half the electron trajectories have a final energy above $1 \mathrm{GeV}$ and electrons on a few trajectories reach maximum kinetic energies above $3 \mathrm{GeV}$. Also, the spread in energies for any particular angle of ejection is reduced.

While the Monte Carlo results presented in Figs. 10 and 11 assume that the target $\mathrm{Fe}^{25+}$ ions are placed at the center of the laser focus, in Fig. 12 we present results for the perhaps more realistic situation that the target ions are distributed within a small spatial volume. Specifically, we assume the ions are randomly distributed within a cylinder having a radius of $10 \mu \mathrm{m}$ and a longitudinal length of $1 \mathrm{~mm}$ along the $z$ axis, centered at the laser focus. The laser parameters are the same as those in Fig. 11; in particular, $w_{0}=20 \mu \mathrm{m}$. The results shown in Fig. 12 for this spatial averaging case indicate that there is no longer even an approximately monochromatic variation of final electron energy versus electron ejection angle, as has been shown also in Ref. [48]. Nevertheless, a significantly large number of electron trajectories still result in final electron kinetic energies above $1 \mathrm{GeV}$.

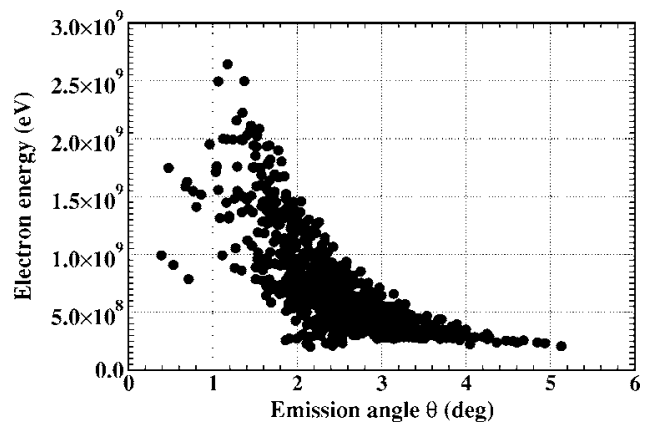

FIG. 12. Spatially averaged Monte Carlo results for the same laser parameters as for the results in Fig. 11. Spatial averaging was carried out by randomly placing target $\mathrm{Fe}^{25+}$ ions inside a cylinder centered at the laser focus and having a radius of $10 \mu \mathrm{m}$ and a longitudinal length of $1 \mathrm{~mm}$. 


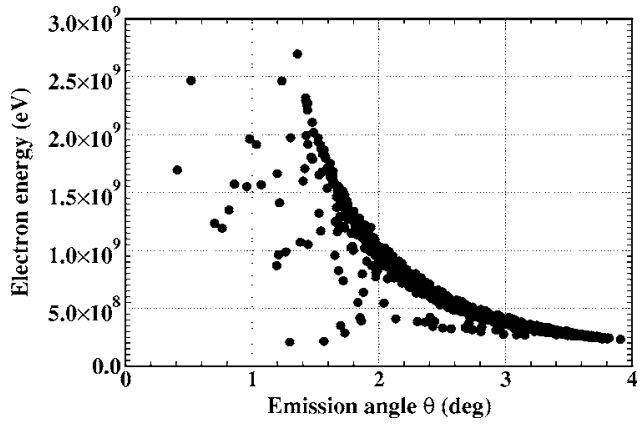

FIG. 13. Monte Carlo results for highly charged $\mathrm{Fe}^{25+}$ ions (located at the laser focus) interacting with an intense laser pulse having the same parameters as for the results in Fig. 11 except for a duration that is 5 times longer-i.e., having 75 cycles, including a 25 -cycle rise time, 25-cycle flat top, and a 25-cycle turn-off. Ionized electron energies are plotted vs their ejection angle $\theta$ with respect to the laser propagation axis.

\section{Dependence of ionized electron energies on laser pulse duration and on the charge of the HCI}

To investigate the effect of laser duration on the energy spectrum of the ionized electrons, we have carried out Monte Carlo calculations using different pulse durations. As an example, Fig. 13 shows results for the case of a 75-cycle laser pulse having a linear turn-on and turn-off of 25 laser cycles each and a 25-cycle flat top. The laser intensity is 2 $\times 10^{22} \mathrm{~W} / \mathrm{cm}^{2}$, the beam waist is $w_{0}=20 \mu \mathrm{m}$, and the wavelength is $1054 \mathrm{~nm}$. These laser parameters are the same as for the short-pulse (15-laser-cycle) case whose results are shown in Fig. 11. Comparing the long-pulse results in Fig. 13 to the short-pulse results in Fig. 11, we see that there is little difference in the spectrum of ionized electron energies despite the factor of 5 difference in laser pulse lengths. Note that to ensure that quantum-mechanical tunneling ionization is insignificant in our classical calculations, we have verified (using the ADK tunneling formula for ions [71]) that the tunneling ionization probability over the rise time of our short pulse is much less than $1 \%$ (it is actually about $0.26 \%$ ); thus, even for the case of a 5-times-longer pulse, the probability of tunneling ionization during the rise time of the pulse is quite small. Hence the present classical prediction of an unchanged ionized electron energy spectrum with increasing pulse duration is expected to be valid. Thus, experiments using HCI's for laser acceleration of electrons appear to be feasible for ultraintense, long laser pulses having durations of the order of $\sim 200 \mathrm{fs}$, which is appropriate for current petawatt laser systems [2].

The other issue of using HCI's for laser acceleration of electrons is the dependence on the charge state of the highly charged ion. To investigate this effect, we carried out a Monte Carlo simulation employing (hydrogenlike) $\mathrm{Co}^{26+}(Z$ $=27$ ) ions as targets instead of $\mathrm{Fe}^{25+}(Z=26)$. Owing to its stronger binding potential, for $\mathrm{Co}^{26+}$ we must apply a more intense laser pulse to ionize the electron initially bound by the ionic core, $\mathrm{Co}^{27+}$. Specifically, we use an ultraintense laser pulse with an intensity of $2.49 \times 10^{22} \mathrm{~W} / \mathrm{cm}^{2}$, a wavelength of $1054 \mathrm{~nm}$, and a pulse duration of 15 laser cycles.

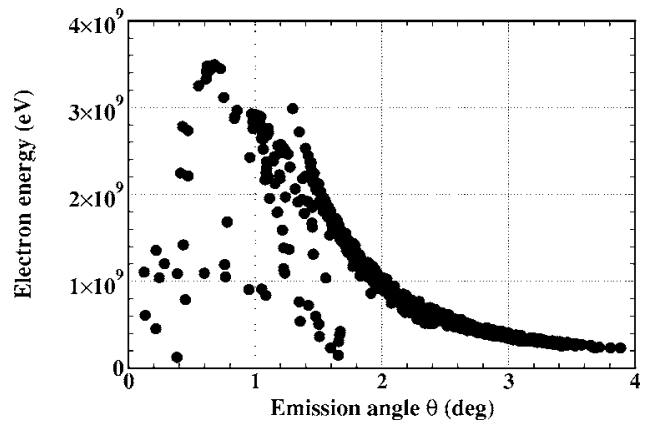

FIG. 14. Monte Carlo results for target ions $\mathrm{Co}^{26+}(Z=27)$ exposed to a laser pulse having a greater intensity of 2.49 $\times 10^{22} \mathrm{~W} / \mathrm{cm}^{2}$ but the same duration $(15$ cycles $)$ and laser beam waist $(20 \mu \mathrm{m})$ as in Fig. 11 for the $\mathrm{Fe}^{25+}$ case (with laser intensity of $2 \times 10^{22} \mathrm{~W} / \mathrm{cm}^{2}$ ). We see that the distribution of electron energies is quite similar to that shown in Fig. 11.

The simulation results are shown in Fig. 14 for the case of a beam waist of $w_{0}=20 \mu \mathrm{m}$. Similar to the situation considered in Fig. 11, the ions were placed at the center of the laser focus. Figure 14 indicates that the electron energy distribution is quite similar to that shown in Fig. 11 for the $\mathrm{Fe}^{25+}$ $(Z=26)$ ion target, with the majority of electron trajectories having final-state electron energies above $1 \mathrm{GeV}$ despite the focusing-induced longitudinal field component $E_{z}$.

There appears, therefore, to be substantial flexibility in the choice of the particular HCI used as a target. However, for any particular choice of ion, one must also choose an appropriate laser intensity. In our calculations we have chosen the laser intensity for a given hydrogenic ion (having nuclear charge $Z$ ) in such a way that the ionization probability during the rise time of the pulse is less than $1 \%$. A rough scaling law can be estimated as follows: the ADK tunneling ionization rate (averaged over a laser period) is

$$
R(Z, I)=\sqrt{\frac{12}{\pi^{3}}} \sqrt{\frac{Z^{7}}{F}} \exp \left(-\frac{2 Z^{3}}{3 F}\right)
$$

where the laser electric field amplitude $F$ is in atomic units (a.u.) and is related to the laser intensity $I$ by

$$
F(\text { a.u. })=5.338 \times 10^{-9} \sqrt{I\left(\mathrm{~W} / \mathrm{cm}^{2}\right)} .
$$

One sees that in the argument of the exponential, the laser amplitude scales as $Z^{3}$. Thus, when increasing the nuclear charge from $Z=26$ to $Z=27$, the laser intensity must be increased by about $25 \%$ in order to ionize the bound electron when the laser pulse reaches its peak intensity. More accurately, and especially for larger fractional changes in $Z$, the preexponential factor in Eq. (23) must be taken into account in choosing the optimal laser intensity for a given nuclear charge. This approximate scaling of laser intensity with $Z$ is demonstrated in Table I. One sees clearly that by increasing the laser intensity by about $25 \%$ when the nuclear charge is changed from $Z=26$ to $Z=27$, the ADK ionization rates remain approximately the same, both during the five-cycle "ramp" and during the five-cycle flat top of the laser pulse. One sees also that the CTMC ionization probabilities, defined as the percentage of classical trajectories that result in 

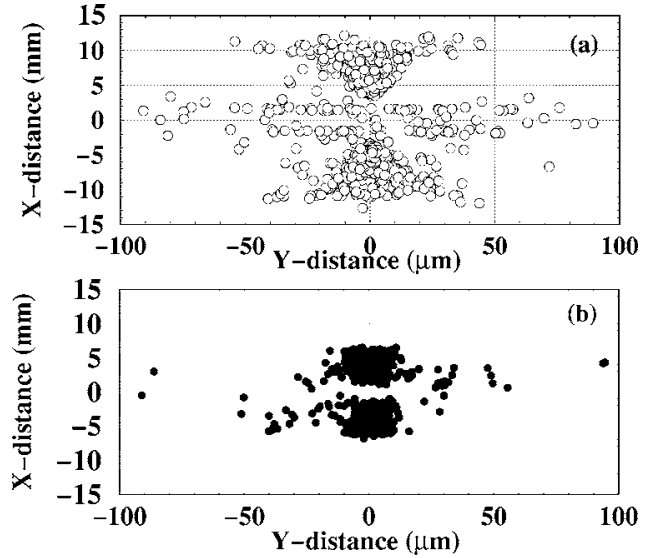

FIG. 15. Spatial distribution of ionized electrons in a detection plane perpendicular to the laser propagation direction (along the $z$ axis) and located $10 \mathrm{~cm}$ from the center of the laser focus. (a) Results for the ionized electron distribution shown in Fig. 10, having $w_{0}=10 \mu \mathrm{m}$. (b) Results for the ionized electron distribution shown in Fig. 11, having $w_{0}=20 \mu \mathrm{m}$.

ionization, also remain approximately the same. Remarkably, one sees that the CTMC ionization probabilities are in reasonable (i.e., within a factor of 2) agreement with the quantum ADK estimates. We emphasize that the analysis presented in this paper concerns the energy and angular distributions of ionized electrons and that the absolute amount of ionization is not essential for these analyses.

Owing to the great sensitivity of the ionization rate on $Z$, we expect that if there are HCI targets of mixed charge states, the laser will selectively ionize only electrons from one of the charge states. For example, if in the laser focal region there are ion charge states $Z=25-27$, we expect that a laser having an intensity of $I=2 \times 10^{22} \mathrm{~W} / \mathrm{cm}^{2}$ will ionize $Z=25$ ions during its turn-on stage (thereby not accelerating them to $\mathrm{GeV}$ energies), will ionize $Z=26$ ions at its peak laser intensity (thereby resulting in efficient acceleration of electrons to $\mathrm{GeV}$ energies), and will not ionize significantly those ions with higher charge states (e.g., $Z=27$ ).

\section{Spatial distribution of ionized electrons}

To estimate the spatial distribution of electrons accelerated by an ultraintense laser pulse, we consider a detecting plane placed perpendicularly to the laser propagation along the $z$ axis and located a distance of $10 \mathrm{~cm}$ from the center of the laser focus. We calculate the expected position on this detection plane (an $x-y$ plane) for each electron trajectory. For the Monte Carlo simulations shown in Figs. 10 and 11, the spatial distributions of the accelerated electrons are plotted in Figs. 15(a) and 15(b), respectively. The results differ only in the laser beam waist, which is $w_{0}=10 \mu \mathrm{m}$ in Fig. 15(a) and $w_{0}=20 \mu \mathrm{m}$ in Fig. 15(b). Note that the distances are shown in units of $\mathrm{mm}$ and $\mu \mathrm{m}$ along the $x$ and $y$ axes, respectively. Since there is no dominant force along the $y$ direction, the ejected electron distribution is very narrow in this dimension. One sees that there are two main groups of electrons, centered along the positive and negative $x$ axis and distributed symmetrically with respect to $x=0$. These two

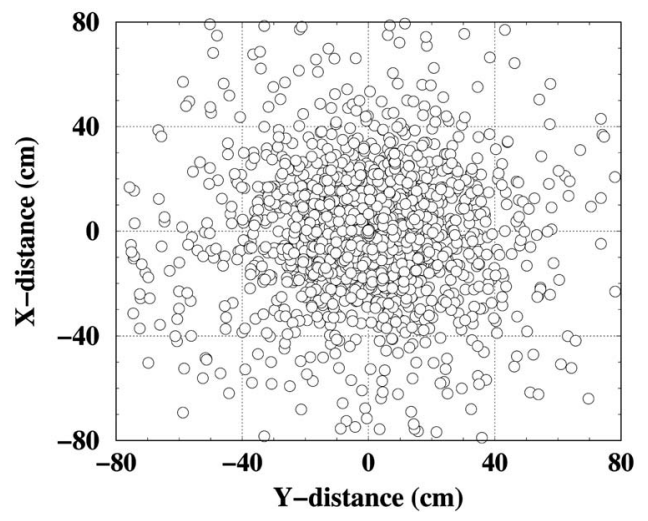

FIG. 16. Effect of spatial averaging of the initial ion distribution (within the laser focal region) on the ionized electron spatial distribution in a detection plane perpendicular to the laser propagation direction (along the $z$ axis) and located $10 \mathrm{~cm}$ from the center of the laser focus. Parameters are the same as for Fig. 15(b), but for the case that the $\mathrm{Fe}^{25+}$ ions are initially distributed within a cylinder centered at the laser focus and having a radius of $\rho=10 \mu \mathrm{m}$ and a longitudinal length of $1 \mathrm{~mm}$.

groups originate from ionization events taking place at the positive or negative laser field maxima (which correspond to the distributions for negative or positive $x$, respectively). Comparing Figs. 15(a) and 15(b), we find that for the larger beam waist $\left(w_{0}=20 \mu \mathrm{m}\right)$ the electron distribution is relatively more compact than for the smaller one $\left(w_{0}=10 \mu \mathrm{m}\right)$. This fact is a result of the dependence of the longitudinal field $E_{z}$ on the laser beam waist [cf. Eq. (18)]. The larger $w_{0}$, the smaller $E_{z}$, and hence the less distortion of the electron spatial distribution.

Note that the results shown in Figs. 15(a) and 15(b) are for the case in which the ions are assumed to be placed initially at the center of the laser focus. More realistically, the target ions would be distributed within a spatial volume centered about the laser focus. Taking into account this spatial averaging, whose effect on the ionized electron energy distribution is shown in Fig. 12 for a beam waist of $w_{0}$ $=20 \mu \mathrm{m}$, we obtain the spatial distribution of ionized electrons shown in Fig. 16. Our results correspond to those shown in Fig. 15(b), except that here the ions are assumed to be randomly distributed within a cylinder having a radius of $\rho=10 \mu \mathrm{m}$ and a longitudinal length of $1 \mathrm{~mm}$. One sees that the electron distribution is very extended (with distances measured in $\mathrm{cm}$ ) along both the $x$ and $y$ axes, although the peak electron density is along the laser propagation direction (i.e., $x=y=0$ ). Comparing Figs. 16 and 12 we conclude that, even for ion targets distributed about the laser focal region, the ionized electrons having the highest energies have the smallest angular spread about the $z$ axis so that collimating the ionized electrons will not only select the most energetic ones but also produce the smallest spatial distribution about the $z$ axis.

\section{SUMMARY AND CONCLUSIONS}

We have used a three-dimensional classical trajectory Monte Carlo method to investigate electron acceleration by 
means of ultraintense laser pulse interactions with highly charged, hydrogenic ions. We have analyzed the dynamics of laser acceleration of the electrons in detail. We find that highly charged ions are good candidates as electron sources for ultraintense laser acceleration owing to the fact that their bound electrons are so tightly bound within the highly charged ionic cores that they are able to remain bound during the turn-on duration of the laser pulse. When such electrons are ionized at the peak laser intensity, they are quickly accelerated along the laser propagation axis by the huge laser Lorentz force, $\mathbf{v} \times \mathbf{B}$. Therefore, these electrons can "surf" on the propagating laser wave and continue being accelerated to energies of the order of GeV. Furthermore, tight laser focusing effects have been analyzed in detail. Our CTMC simulations demonstrate that the tight-focusing-induced longitudinal field $E_{z}$ serves to destroy the monochromatic relation between ionized electron energy and electron ejection angle. Nevertheless, large numbers of $\mathrm{GeV}$ electrons are still produced for the case of tight focusing, and for some trajectories, the ionized electron energies are higher than for the case of loose focusing. Experimentally, highly charged ions may be produced either by employing a laser prepulse $[12,13]$ or by various electronic, atomic, or ionic collision processes [74]. The highest ion densities produced by collision processes can either at present or in the near future reach $10^{9}-10^{11} / \mathrm{cm}^{3}[72,74]$; in the more distant future, ion densities of $10^{14} / \mathrm{cm}^{3}$ may become possible [75]. A double-pulse scheme might also be feasible in which a laser pulse is split into two subpulses, one of which is first used to irradiate a cluster or a solid target, thereby producing highly charged ions, and the other of which may be delayed so that it interacts directly with the highly charged ions that are produced. However, one needs to make sure that the subsequent accelerating laser pulse can penetrate into the ion plasma that is produced. Otherwise, an ion beam source may be required.

\section{ACKNOWLEDGMENTS}

The authors gratefully acknowledge informative discussions with J.D. Gillaspy on ion beam sources. This work was supported in part by the U.S. Department of Energy, Office of Science, Division of Chemical Sciences, Geosciences, and Biosciences under Grant No. DE-FG02-96ER14646.
[1] D. Strickland and G. Mourou, Opt. Commun. 56, 219 (1985); P. Maine, D. Strickland, P. Bado, M. Pessot, and G. Mourou, IEEE J. Quantum Electron. 24, 398 (1988).

[2] M. D. Perry et al., Opt. Lett. 24, 160 (1999).

[3] B. E. Lemoff and C. P. J. Barty, Opt. Lett. 18, 1651 (1993).

[4] M. D. Perry and G. Mourou, Science 264, 917 (1994).

[5] J. Zhou, C. P. Huang, M. M. Murnane, and H. C. Kapteyn, Opt. Lett. 20, 64 (1995).

[6] T. Tajima and G. Mourou, Phys. Rev. ST Accel. Beams 5, 031301 (2002).

[7] S. V. Bulanov, T. Esirkepov, and T. Tajima, Phys. Rev. Lett. 91, 085001 (2003).

[8] G. A. Mourou, C. P. J. Barty, and M. D. Perry, Phys. Today 51(1), 22 (1998).

[9] M. Protopapas, C. H. Keitel, and P. L. Knight, Rep. Prog. Phys. 60, 389 (1997).

[10] T. Brabec and F. Krausz, Rev. Mod. Phys. 72, 545 (2000).

[11] W. Becker, F. Grasbon, R. Kopold, D. B. Milošević, G. G. Paulus, and H. Walther, Adv. At., Mol., Opt. Phys. 42, 35 (2002).

[12] T. Ditmire, T. Donnelly, R. W. Falcone, and M. D. Perry, Phys. Rev. Lett. 75, 3122 (1995).

[13] E. M. Snyder, S. A. Buzza, and A. W. Castleman, Jr., Phys. Rev. Lett. 77, 3347 (1996).

[14] T. Ditmire, J. W. G. Tisch, E. Springate, M. B. Mason, N. Hay, R. A. Smith, J. Marangos, and M. H. R. Hutchinson, Nature (London) 386, 54 (1997).

[15] T. Ditmire, E. Springate, J. W. G. Tisch, Y. L. Shao, M. B. Mason, N. Hay, J. P. Marangos, and M. H. R. Hutchinson, Phys. Rev. A 57, 369 (1998).

[16] E. L. Clark, K. Krushelnick, J. R. Davies, M. Zepf, M. Tatarakis, F. N. Beg, A. Machacek, P. A. Norreys, M. I. K. Santala, I. Watts, and A. E. Dangor, Phys. Rev. Lett. 84, 670 (2000).
[17] A. Maksimchuk, S. Gu, K. Flippo, D. Umstadter, and V. Y. Bychenkov, Phys. Rev. Lett. 84, 4108 (2000).

[18] M. J. Feldman and R. Y. Chiao, Phys. Rev. A 4, 352 (1971).

[19] T. Tajima and J. M. Dawson, Phys. Rev. Lett. 43, 267 (1979).

[20] E. Esarey, P. Sprangle, J. Krall, and A. Ting, IEEE Trans. Plasma Sci. 24, 252 (1996).

[21] C. Joshi, T. Tajima, J. M. Dawson, H. A. Baldis, and N. A. Ebrahim, Phys. Rev. Lett. 47, 1285 (1981).

[22] J. B. Rosenzweig, D. B. Cline, B. Cole, H. Figueroa, W. Gai, R. Konecny, J. Norem, P. Schoessow, and J. Simpson, Phys. Rev. Lett. 61, 98 (1988).

[23] Y. Kitagawa, T. Matsumoto, T. Minamihata, K. Sawai, K. Matsuo, K. Mima, K. Nishihara, H. Azechi, K. A. Tanaka, H. Takabe, and S. Nakai, Phys. Rev. Lett. 68, 48 (1992).

[24] C. E. Clayton, K. A. Marsh, A. Dyson, M. Everett, A. Lal, W. P. Leemans, R. Williams, and C. Joshi, Phys. Rev. Lett. 70, 37 (1993).

[25] K. Nakajima et al., Phys. Rev. Lett. 74, 4428 (1995).

[26] F. Amiranoff, D. Bernard, B. Cros, F. Jacquet, G. Matthieussent, P. Miné, P. Mora, J. Morillo, F. Moulin, A. E. Specka, and C. Stenz, Phys. Rev. Lett. 74, 5220 (1995).

[27] F. Dorchies et al., Phys. Plasmas 6, 2903 (1999).

[28] X. Wang, M. Krishnan, N. Saleh, H. Wang, and D. Umstadter, Phys. Rev. Lett. 84, 5324 (2000).

[29] S. P. D. Mangles, C. D. Murphy, Z. Najmudin, A. G. R. Thomas, J. L. Collier, A. E. Dangor, E. J. Divall, P. S. Foster, J. G. Gallacher, C. J. Hooker, D. A. Jaroszynski, A. J. Langley, W. B. Mori, P. A. Norreys, F. S. Tsung, R. Viskup, B. R. Walton, and K. Krushelnick, Nature (London) 431, 535 (2004).

[30] C. G. R. Geddes, Cs. Toth, J. van Tilborg, E. Esarey, C. B. Schroeder, D. Bruhwiler, C. Nieter, J. Cary, and W. P. Leemans, Nature (London) 431, 538 (2004).

[31] J. Faure, Y. Glinec, A. Pukhov, S. Kiselev, S. Gordienko, E. 
Lefebvre, J.-P. Rousseau, F. Burgy, and V. Malka, Nature (London) 431, 541 (2004).

[32] P. Sprangle, B. Hafizi, J. R. Peñano, R. F. Hubbard, A. Ting, A. Zigler, and T. M. Antonsen, Jr., Phys. Rev. Lett. 85, 5110 (2000).

[33] D. Umstadter, J. K. Kim, and E. Dodd, Phys. Rev. Lett. 76, 2073 (1996).

[34] C. I. Moore, A. Ting, S. J. McNaught, J. Qiu, H. R. Burris, and P. Sprangle, Phys. Rev. Lett. 82, 1688 (1999).

[35] D. Umstadter, J. Phys. D 36, R151 (2003).

[36] F. V. Hartemann, S. N. Fochs, G. P. Le Sage, N. C. Luhmann, Jr., J. G. Woodworth, M. D. Perry, and Y. J. Chen, and A. K. Kerman, Phys. Rev. E 51, 4833 (1995).

[37] B. Quesnel and P. Mora, Phys. Rev. E 58, 3719 (1998).

[38] J. X. Wang, Y. K. Ho, L. Feng, Q. Kong, P. X. Wang, Z. S. Yuan, and W. Scheid, Phys. Rev. E 60, 7473 (1999).

[39] Y. I. Salamin and F. H. M. Faisal, Phys. Rev. A 61, 043801 (2000); Y. I. Salamin, F. H. M. Faisal, and C. H. Keitel, Phys. Rev. A 62, 053809 (2000).

[40] C. I. Moore, J. P. Knauer, and D. D. Meyerhofer, Phys. Rev. Lett. 74, 2439 (1995).

[41] G. Malka, E. Lefebvre, and J. L. Miquel, Phys. Rev. Lett. 78, 3314 (1997).

[42] S. X. Hu and A. F. Starace, Phys. Rev. Lett. 88, 245003 (2002)

[43] W. D. Kimura et al., Phys. Rev. Lett. 86, 4041 (2001).

[44] Y. I. Salamin and C. H. Keitel, Phys. Rev. Lett. 88, 095005 (2002).

[45] Y. I. Salamin, G. R. Mocken, and C. H. Keitel, Phys. Rev. E 67, 016501 (2003).

[46] C. H. Keitel, J. Phys. B 29, L873 (1996).

[47] Y. L. Shao, T. Ditmire, J. W. G. Tisch, E. Springate, J. P. Marangos, and M. H. R. Hutchinson, Phys. Rev. Lett. 77, 3343 (1996).

[48] A. Maltsev and T. Ditmire, Phys. Rev. Lett. 90, 053002 (2003).

[49] M. Lax, Phys. Rev. A 11, 1365 (1975).

[50] L. W. Davis, Phys. Rev. A 19, 1177 (1979).

[51] G. P. Agrawal and D. N. Pattanayak, J. Opt. Soc. Am. 69, 575 (1979).

[52] J. P. Barton and D. R. Alexander, J. Appl. Phys. 66, 2800
(1989).

[53] M. Casu, C. Szymanowski, S. X. Hu, and C. H. Keitel, J. Phys. B 33, L411 (2000).

[54] S. X. Hu and C. H. Keitel, Phys. Rev. A 63, 053402 (2001).

[55] S. X. Hu, D. B. Milošević, W. Becker, and W. Sandner, Phys. Rev. A 64, 013410 (2001).

[56] N. J. Kylstra, R. M. Potvliege, and C. J. Joachain, J. Phys. B 34, L55 (2001); Laser Phys. 12, 409 (2002).

[57] M. Casu and C. H. Keitel, Europhys. Lett. 58, 496 (2002).

[58] N. Milosevic, V. P. Krainov, and T. Brabec, Phys. Rev. Lett. 89, 193001 (2002).

[59] S. X. Hu, A. F. Starace, W. Becker, W. Sandner, and D. B. Milošević, J. Phys. B 35, 627 (2002).

[60] J. Chen, J. Liu, L. B. Fu, and W. M. Zheng, Phys. Rev. A 63, 011404(R) (2001).

[61] J. G. Leopold and I. C. Percival, Phys. Rev. Lett. 41, 944 (1978); J. Phys. B 12, 709 (1979).

[62] S.-I. Chu and R. Y. Yin, J. Opt. Soc. Am. B 4, 720 (1987).

[63] G. A. Kyrala, J. Opt. Soc. Am. B 4, 731 (1987).

[64] M. Gajda, J. Grochmalicki, M. Lewenstein, and K. Rzażewski, Phys. Rev. A 46, 1638 (1992).

[65] H. Schmitz, K. Boucke, and H.-J. Kull, Phys. Rev. A 57, 467 (1998).

[66] L. N. Gaier and C. H. Keitel, Phys. Rev. A 65, 023406 (2002).

[67] R. D. Sard, Relativistic Mechanics (Benjamin, New York, 1970), p. 189.

[68] J. S. Cohen, Phys. Rev. A 26, 3008 (1982).

[69] R. Abrines and I. C. Percival, Proc. Phys. Soc. London 88, 861 (1966); 88, 873 (1966).

[70] R. E. Olson and A. Salop, Phys. Rev. A 16, 531 (1977).

[71] M. V. Ammosov, N. B. Delone, and V. P. Krainov, Zh. Eksp. Teor. Fiz. 91, 2008 (1986) [Sov. Phys. JETP 64, 1191 (1986)].

[72] J. D. Gillaspy, J. Phys. B 34, R93 (2001).

[73] J. D. Jackson, Classical Electrodynamics, 3rd ed. (Wiley, New York, 1999), Eq. (14.26).

[74] P. H. Mokler and Th. Stoehlker, Adv. At., Mol., Opt. Phys. 37, 297 (1996); J. Ullrich, R. Moshammer, R. Dorner, O. Jagutzki, V. Mergel, H. SchmidtBocking, and L. Spielberger, J. Phys. B 30, 2917 (1997).

[75] J. D. Gillaspy (private communication). 